\title{
A strong convergence theorem for Bregman asymptotically quasi-nonexpansive mappings in the intermediate sense
}

\section{Yukino Tomizawa*}

\author{
"Correspondence: \\ tomizawa@gug.math.chuo-u.ac.jp \\ Department of Mathematics, \\ Graduate School of Science and \\ Engineering, Chuo University, \\ 1-13-27 Kasuga, Bunkyo-ku, Tokyo, \\ 112-8551, Japan \\ Center for Fundamental Science, \\ Kaohsiung Medical University, 100, \\ Shih-Chuan 1st Road, Kaohsiung, \\ 807, Taiwan
}

\begin{abstract}
The purpose of this paper is to introduce a new class of Bregman asymptotically quasi-nonexpansive mappings in the intermediate sense. A strong convergence theorem of the shrinking projection method with the modified Mann iteration is established to find fixed points of the mappings in reflexive Banach spaces. This theorem generalizes some known results in the current literature.
\end{abstract}

MSC: 47H09; 47J25

Keywords: asymptotically quasi-nonexpansive in the intermediate sense; Bregman distance; Bregman projection; Legendre function; totally convex function

\section{Introduction}

Fixed point theory is an important branch of nonlinear analysis and has been applied in numerous studies of nonlinear phenomena. Many problems in nonlinear functional analysis is related to finding fixed points of nonlinear mappings of nonexpansive type. From the standpoint of real world applications, we want to construct an iterative process to approximate fixed points of mappings of nonexpansive type. Many authors have considered the problem of iterative algorithms for mappings of nonexpansive type which converge to some fixed points.

Let $C$ be a nonempty subset of a real Banach space and $T$ a nonlinear mapping from $C$ into itself. We denote by $F(T)$ the set of fixed points of $T$. Recall that $T$ is said to be nonexpansive if

$$
\|T x-T y\| \leq\|x-y\| \quad \text { for all } x, y \in C \text {. }
$$

More generally, $T$ is said to be asymptotically nonexpansive (cf. [1]) if there exists a sequence $\left\{k_{n}\right\} \subset[1, \infty)$ with $\lim _{n \rightarrow \infty} k_{n}=1$ such that

$$
\left\|T^{n} x-T^{n} y\right\| \leq k_{n}\|x-y\| \quad \text { for all } x, y \in C \text { and } n \geq 1 .
$$

In the framework of Hilbert spaces, Takahashi, Takeuchi and Kubota [2] have introduced a new hybrid iterative scheme called a shrinking projection method for nonexpansive mappings. It is an advantage of projection methods that the strong convergence of iterative

\section{Springer}

C2014 Tomizawa; licensee Springer. This is an Open Access article distributed under the terms of the Creative Commons Attribution License (http://creativecommons.org/licenses/by/2.0), which permits unrestricted use, distribution, and reproduction in any medium, provided the original work is properly cited. 
sequences is guaranteed without any compact assumptions. Moreover, Schu [3] has introduced a modified Mann iteration to approximate fixed points of asymptotically nonexpansive mappings in uniformly convex Banach spaces. Motivated by [2, 3], Inchan [4] has introduced a new hybrid iterative scheme by using the shrinking projection method with the modified Mann iteration for asymptotically nonexpansive mappings. The mapping $T$ is said to be asymptotically nonexpansive in the intermediate sense (cf. [5]) if

$$
\limsup _{n \rightarrow \infty} \sup _{x, y \in C}\left(\left\|T^{n} x-T^{n} y\right\|-\|x-y\|\right) \leq 0 .
$$

If $F(T)$ is nonempty and (1.1) holds for all $x \in C$ and $y \in F(T)$, then $T$ is said to be asymptotically quasi-nonexpansive in the intermediate sense. It is worth mentioning that the class of asymptotically nonexpansive mappings in the intermediate sense contains properly the class of asymptotically nonexpansive mappings, since the mappings in the intermediate sense are not Lipschitz continuous in general.

Recently, many authors have studied further new hybrid iterative schemes in the framework of real Banach spaces; for instance, see [6-8]. Qin and Wang [9] have introduced a new class of mappings which are asymptotically quasi-nonexpansive with respect to the Lyapunov functional (cf. [10]) in the intermediate sense. By using the shrinking projection method, Hao [11] has proved a strong convergence theorem for an asymptotically quasi-nonexpansive mapping with respect to the Lyapunov functional in the intermediate sense.

In 1967, Bregman [12] has discovered an elegant and effective technique for the using of the so-called Bregman distance function (see Section 2) in the process of designing and analyzing feasibility and optimization algorithms. This opened a growing area of research in which Bregman's technique is applied in various ways in order to design and analyze not only iterative algorithms for solving feasibility and optimization problems, but also algorithms for solving variational inequalities, for approximating equilibria and for computing fixed points of nonlinear mappings.

The purpose of this paper is to prove strong convergence theorems for asymptotically quasi-nonexpansive mappings with respect to Bregman distances in the intermediate sense by using the shrinking projection method. Many authors have studied iterative methods for approximating fixed points of mappings of nonexpansive type with respect to the Bregman distance; see [13-16]. However, nonlinear mappings which are not Lipschitz continuous with respect to the Bregman distance have not been studied yet. Against this background, we introduce a new class of asymptotically quasi-nonexpansive mappings which is an extension of with respect to the Bregman distance in the intermediate sense. Motivated by the results above, we design a new hybrid iterative scheme for finding fixed points of the mapping in reflexive Banach spaces. This iterative method is expected to be applicable to many other problems in nonlinear functional analysis relating to Bregman distances.

In this paper, we introduce a new class of nonlinear mappings which is an extension of asymptotically quasi-nonexpansive mappings with respect to the Bregman distance in the intermediate sense. Motivated by $[4,12]$, we design a new hybrid iterative scheme for finding a fixed point of mappings in the new class by using the shrinking projection method with respect to Bregman distances in reflexive Banach spaces. We prove a new strong convergence theorem for the mappings, which is an extension of results of [11]. In Section 2, 
we present several preliminary definitions and results. In Section 3, we introduce the new class of mappings with respect to the Bregman distance and prove closedness and convexity of the set of fixed points of the mappings. In Section 4, we prove a strong convergence theorem for finding a fixed point of mappings in the new class by using the shrinking projection method.

\section{Preliminaries}

Throughout this paper, we denote by $\mathbf{N}$ and $\mathbf{R}$ the sets of all nonnegative integers and real numbers, respectively, and we assume that $E$ is a real reflexive Banach space with the norm $\|\cdot\|, E^{*}$ the dual space of $E$ and $\langle\cdot, \cdot\rangle$ the pairing between $E$ and $E^{*}$. When $\left\{x_{n}\right\}$ is a sequence in $E$, we denote the strong convergence of $\left\{x_{n}\right\}$ to $x$ by $x_{n} \rightarrow x$ and the weak convergence by $x_{n} \rightarrow x$.

Let $f: E \rightarrow(-\infty,+\infty]$ be a function. The effective domain of $f$ is defined by

$$
\operatorname{dom} f:=\{x \in E: f(x)<+\infty\} .
$$

When $\operatorname{dom} f \neq \emptyset$ we say that $f$ is proper. We denote by int $\operatorname{dom} f$ the interior of the effective domain of $f$. We denote by $\operatorname{ran} f$ the range of $f$.

The function $f$ is said to be strongly coercive if $\lim _{\|x\| \rightarrow \infty} f(x) /\|x\|=+\infty$. Given a proper and convex function $f: E \rightarrow(-\infty,+\infty]$, the subdifferential of $f$ is a mapping $\partial f: E \rightarrow 2^{E^{*}}$ defined by

$$
\partial f(x):=\left\{x^{*} \in E^{*}: f(y) \geq f(x)+\left\langle x^{*}, y-x\right\rangle, \forall y \in E\right\} \quad \text { for all } x \in E .
$$

The Fenchel conjugate function of $f$ is the convex function $f^{*}: E^{*} \rightarrow(-\infty,+\infty)$ defined by

$$
f^{*}(\xi):=\sup \{\langle\xi, x\rangle-f(x): x \in E\}
$$

We know that $x^{*} \in \partial f(x)$ if and only if $f(x)+f^{*}\left(x^{*}\right)=\left\langle x^{*}, x\right\rangle$ for $x \in E$; see [17].

Proposition 2.1 ([18], Proposition 2.47) Let $f: E \rightarrow(-\infty,+\infty]$ be a proper, convex and lower semicontinuous function. Then the following conditions are equivalent:

(i) $\operatorname{ran} \partial f=E^{*}$ and $\partial f^{*}=(\partial f)^{-1}$ is bounded on bounded subsets of $E^{*}$;

(ii) $f$ is strongly coercive.

Let $f: E \rightarrow(-\infty,+\infty]$ be a convex function and $x \in \operatorname{int} \operatorname{dom} f$. For any $y \in E$, we define the right-hand derivative of $f$ at $x$ in the direction $y$ by

$$
f^{\circ}(x, y):=\lim _{t \searrow 0} \frac{f(x+t y)-f(x)}{t} .
$$

The function $f$ is said to be Gâteaux differentiable at $x$ if the limit (2.1) exists for any $y$. In this case, the gradient of $f$ at $x$ is the function $\nabla f(x): E \rightarrow(-\infty,+\infty)$ defined by $\langle\nabla f(x), y\rangle=f^{\circ}(x, y)$ for all $y \in E$. The function $f$ is said to be Gâteaux differentiable if it is Gâteaux differentiable at each $x \in \operatorname{int} \operatorname{dom} f$. If the limit (2.1) is attained uniformly in $\|y\|=1$, then the function $f$ is said to be Fréchet differentiable at $x$. The function $f$ is said 
to be uniformly Fréchet differentiable on a subset $C$ of $E$ if the limit (2.1) is attained uniformly for $x \in C$ and $\|y\|=1$. We know that if $f$ is uniformly Fréchet differentiable on bounded subsets of $E$, then $f$ is uniformly continuous on bounded subsets of $E$ ( $c f$. [19]). We will need the following results.

Proposition 2.2 ([20], Proposition 2.1) If a function $f: E \rightarrow \mathbf{R}$ is convex, uniformly Fréchet differentiable and bounded on bounded subsets of $E$, then $\nabla f$ is uniformly continuous on bounded subsets of $E$ from the strong topology of $E$ to the strong topology of $E^{*}$.

Proposition 2.3 ([21], Proposition 3.6.4) Let $f: E \rightarrow \mathbf{R}$ be a convex function which is bounded on bounded subsets of $E$. Then the following assertions are equivalent:

(i) $f$ is strongly coercive and uniformly convex on bounded subsets of $E$;

(ii) $f^{*}$ is Fréchet differentiable and $\nabla f^{*}$ is uniformly norm-to-norm continuous on bounded subsets of $\operatorname{dom} f^{*}=E^{*}$.

A function $f: E \rightarrow(-\infty,+\infty]$ is said to be admissible if it is proper, convex, and lower semicontinuous on $E$ and Gâteaux differentiable on int $\operatorname{dom} f$. Under these conditions we know that $f$ is continuous in int $\operatorname{dom} f, \partial f$ is single-valued and $\partial f=\nabla f$; see $[17,22]$. An admissible function $f: E \rightarrow(-\infty,+\infty$ ] is called Legendre ( $c f$. [17]) if it satisfies the following two conditions:

(L1) the interior of the domain of $f$, int $\operatorname{dom} f$, is nonempty, $f$ is Gâteaux differentiable and $\operatorname{dom} \nabla f=\operatorname{int} \operatorname{dom} f$;

(L2) the interior of the domain of $f^{*}$, int $\operatorname{dom} f^{*}$, is nonempty, $f^{*}$ is Gâteaux differentiable and $\operatorname{dom} \nabla f^{*}=\operatorname{int} \operatorname{dom} f^{*}$.

Let $f$ be a Legendre function on $E$. Since $E$ is reflexive, we always have $\nabla f=\left(\nabla f^{*}\right)^{-1}$. This fact, when combined with conditions (L1) and (L2), implies the following equalities:

$$
\operatorname{ran} \nabla f=\operatorname{dom} f^{*}=\operatorname{int} \operatorname{dom} f^{*} \quad \text { and } \quad \operatorname{ran} \nabla f^{*}=\operatorname{dom} f=\operatorname{int} \operatorname{dom} f .
$$

Conditions (L1) and (L2) imply that the functions $f$ and $f^{*}$ are strictly convex on the interior of their respective domains.

Example 2.4 The following functions are Legendre on $E=\mathbf{R}^{n}$ : Let $x \in \mathbf{R}^{n}$.

(i) Halved energy: $f(x)=\|x\|^{2} / 2=\frac{1}{2} \sum_{j=1}^{n} x_{j}^{2}$.

(ii) Boltzmann-Shannon entropy:

$$
f(x)= \begin{cases}\sum_{j=1}^{n}\left(x_{j} \ln \left(x_{j}\right)-x_{j}\right), & x \geq 0 ; \\ +\infty, & \text { otherwise }\end{cases}
$$

(iii) Burg entropy:

$$
f(x)= \begin{cases}-\sum_{j=1}^{n} \ln \left(x_{j}\right), & x>0 \\ +\infty, & \text { otherwise }\end{cases}
$$

Note that int $\operatorname{dom} f=\mathbf{R}^{n}$ in (i), whereas int $\operatorname{dom} f=\left\{x \in \mathbf{R}^{n}: x_{j}>0, j=1, \ldots, n\right\}$ in (ii) and (iii). 
Let $f: E \rightarrow(-\infty,+\infty]$ be a convex function on $E$ which is Gâteaux differentiable on int $\operatorname{dom} f$. The bifunction $D_{f}: \operatorname{dom} f \times \operatorname{int} \operatorname{dom} f \rightarrow[0,+\infty)$ given by

$$
D_{f}(y, x):=f(y)-f(x)-\langle\nabla f(x), y-x\rangle
$$

is called the Bregman distance with respect to $f(c f$. [23]). In general, the Bregman distance is not a metric, since it is not symmetric and does not satisfy the triangle inequality. However, it has the following important property, which is called the three point identity (cf. [24]): for any $x \in \operatorname{dom} f$ and $y, z \in \operatorname{int} \operatorname{dom} f$,

$$
D_{f}(x, y)+D_{f}(y, z)-D_{f}(x, z)=\langle\nabla f(z)-\nabla f(y), x-y\rangle .
$$

Example 2.5 The Bregman distances corresponding to the Legendre functions of Example 2.4 are as follows $\left(x, y \in \mathbf{R}^{n}\right)$ :

(i) Euclidean distance: $D_{f}(y, x)=\|y-x\|^{2} / 2$.

(ii) Kullback-Leibler divergence: $D_{f}(y, x)=\sum_{j=1}^{n}\left(y_{j} \ln \left(y_{j} / x_{j}\right)-y_{j}+x_{j}\right)$.

(iii) Itakura-Saito divergence: $D_{f}(y, x)=\sum_{j=1}^{n}\left(\ln \left(x_{j} / y_{j}\right)+y_{j} / x_{j}-1\right)$.

With a Legendre function $f: E \rightarrow(-\infty,+\infty]$, we associate the bifunction $W^{f}: \operatorname{dom} f^{*} \times$ $\operatorname{dom} f \rightarrow[0,+\infty)$ defined by

$$
W^{f}(\xi, x):=f(x)-\langle\xi, x\rangle+f^{*}(\xi) \quad \text { for } x \in \operatorname{dom} f \text { and } \xi \in \operatorname{dom} f^{*}
$$

Proposition 2.6 ([13], Proposition 10) Let $f: E \rightarrow(-\infty,+\infty]$ be a Legendre function such that $\nabla f^{*}$ is bounded on bounded subsets of int $\operatorname{dom} f^{*}$. Let $x \in \operatorname{int} \operatorname{dom} f$. If the sequence $\left\{D_{f}\left(x, x_{n}\right)\right\}_{n \in \mathbf{N}}$ is bounded, then the sequence $\left\{x_{n}\right\}_{n \in \mathbf{N}}$ is also bounded.

Proposition 2.7 ([13], Proposition 1) Let $f: E \rightarrow(-\infty,+\infty]$ be a Legendre function. Then the following statements hold:

(i) The function $W^{f}(\cdot, x)$ is convex for all $x \in \operatorname{dom} f$;

(ii) $W^{f}(\nabla f(x), y)=D_{f}(y, x)$ for all $x \in \operatorname{int} \operatorname{dom} f$ and $y \in \operatorname{dom} f$.

Let $f: E \rightarrow(-\infty,+\infty]$ be a convex function on $E$ which is Gâteaux differentiable on int $\operatorname{dom} f$. The function $f$ is said to be totally convex at a point $x \in \operatorname{int} \operatorname{dom} f$ if its modulus of total convexity at $x, v_{f}(x, \cdot):[0,+\infty) \rightarrow[0,+\infty]$, defined by

$$
v_{f}(x, t):=\inf \left\{D_{f}(y, x): y \in \operatorname{dom} f,\|y-x\|=t\right\},
$$

is positive whenever $t>0$. The function $f$ is said to be totally convex when it is totally convex at every point of int $\operatorname{dom} f$. The function $f$ is said to be totally convex on bounded sets if, for any nonempty bounded set $B \subset E$, the modulus of total convexity of $f$ on $B$, $v_{f}(B, t)$ is positive for any $t>0$, where $v_{f}(B, \cdot):[0,+\infty) \rightarrow[0,+\infty]$ is defined by

$$
v_{f}(B, t):=\inf \left\{v_{f}(x, t): x \in B \cap \operatorname{int} \operatorname{dom} f\right\} .
$$

We remark in passing that $f$ is totally convex on bounded sets if and only if $f$ is uniformly convex on bounded sets; see $[25,26]$. 
Proposition 2.8 ([25], Proposition 4.2) Let $f: E \rightarrow(-\infty,+\infty]$ be a convex function whose domain contains at least two points. Iff is lower semicontinuous, then $f$ is totally convex on bounded sets if and only iff is uniformly convex on bounded sets.

Proposition 2.9 ([27], Lemma 3.1) Let $f: E \rightarrow \mathbf{R}$ be a totally convex function. If $x \in E$ and the sequence $\left\{D_{f}\left(x_{n}, x\right)\right\}_{n \in \mathbf{N}}$ is bounded, then the sequence $\left\{x_{n}\right\}_{n \in \mathbf{N}}$ is also bounded.

Let $f: E \rightarrow(-\infty,+\infty]$ be a convex function on $E$ which is Gâteaux differentiable on int $\operatorname{dom} f$. The function $f$ is said to be sequentially consistent (cf. [26]) if for any two sequences $\left\{x_{n}\right\}_{n \in \mathbf{N}}$ and $\left\{y_{n}\right\}_{n \in \mathbf{N}}$ in int $\operatorname{dom} f$ and $\operatorname{dom} f$, respectively, such that the first one is bounded,

$$
\lim _{n \rightarrow \infty} D_{f}\left(y_{n}, x_{n}\right)=0 \quad \text { implies } \quad \lim _{n \rightarrow \infty}\left\|y_{n}-x_{n}\right\|=0 .
$$

Proposition 2.10 ([22], Proposition 2.1.2) A function $f: E \rightarrow(-\infty,+\infty]$ is totally convex on bounded subsets of $E$ if and only if it is sequentially consistent.

Let $C$ be a nonempty, closed, and convex subset of $E$. Let $f: E \rightarrow(-\infty,+\infty]$ be a convex function on $E$ which is Gâteaux differentiable on int $\operatorname{dom} f$. The Bregman projection $\operatorname{proj}_{C}^{f}(x)$ with respect to $f$ (cf. [23]) of $x \in \operatorname{int} \operatorname{dom} f$ onto $C$ is the minimizer over $C$ of the functional $D_{f}(\cdot, x): E \rightarrow[0,+\infty]$, that is,

$$
\operatorname{proj}_{C}^{f}(x):=\arg \min \left\{D_{f}(y, x): y \in C\right\} .
$$

Proposition 2.11 ([28], Corollary 2.1) Let $f: E \rightarrow \mathbf{R}$ be an admissible, strongly coercive, and strictly convex function. Let $C$ be a nonempty, closed, and convex subset of $\operatorname{dom} f$. Then $\operatorname{proj}_{C}^{f}(x)$ exists uniquely for all $x \in \operatorname{int} \operatorname{dom} f$.

Remark Let $f(x)=\|x\|^{2} / 2$ for $x \in E$.

(i) If $E$ is a Hilbert space, then the Bregman projection $\operatorname{proj}_{C}^{f}$ is reduced to the metric projection onto $C$.

(ii) If $E$ is a smooth Banach space, then the Bregman projection $\operatorname{proj}_{C}^{f}$ is reduced to the generalized projection $\Pi_{C} x$ which is defined by

$$
\Pi_{C} x:=\arg \min \{\phi(y, x): y \in C\},
$$

where $\phi: E \times E \rightarrow \mathbf{R}^{+}$is the Lyapunov functional (cf. [10]) defined by $\phi(y, x):=\|y\|^{2}-2\langle y, J x\rangle+\|x\|^{2}$ for all $x, y \in E$.

Proposition 2.12 ([26], Corollary 4.4) Let $f: E \rightarrow(-\infty,+\infty]$ be a totally convex function. Let $C$ be a nonempty, closed, and convex subset of int $\operatorname{dom} f$ and $x \in \operatorname{int} \operatorname{dom} f$. If $\hat{x} \in C$, then the following statements are equivalent:

(i) The vector $\hat{x}$ is the Bregman projection $\operatorname{proj}_{C}^{f}(x)$ of $x$ onto $C$.

(ii) The vector $\hat{x}$ is the unique solution $z$ of the variational inequality

$$
\langle\nabla f(x)-\nabla f(z), z-y\rangle \geq 0 \quad \text { for all } y \in C .
$$


(iii) The vector $\hat{x}$ is the unique solution $z$ of the inequality

$$
D_{f}(y, z)+D_{f}(z, x) \leq D_{f}(y, x) \quad \text { for all } y \in C \text {. }
$$

\section{Bregman asymptotically quasi-nonexpansive in the intermediate sense}

Let $C$ be a nonempty, closed, and convex subset of $E$ and $T$ a mapping from $C$ into itself. Let $f: E \rightarrow(-\infty,+\infty]$ be an admissible function. Recall that the mapping $T$ is said to be Bregman quasi-nonexpansive (cf. [14]) if $F(T) \neq \emptyset$ and

$$
D_{f}(p, T x) \leq D_{f}(p, x) \quad \text { for all } p \in F(T) \text { and } x \in C .
$$

The mapping $T$ is said to be Bregman asymptotically quasi-nonexpansive (cf. [29]) if $F(T) \neq \emptyset$ and there exists a sequence $\left\{k_{n}\right\} \subset[1, \infty)$ with $\lim _{n \rightarrow \infty} k_{n}=1$ such that

$$
D_{f}\left(p, T^{n} x\right) \leq k_{n} D_{f}(p, x) \quad \text { for all } p \in F(T), x \in C \text { and } n \in \mathbf{N} \text {. }
$$

Every Bregman quasi-nonexpansive mapping is Bregman asymptotically quasi-nonexpansive with $k_{n}=1$.

We introduce a new class of mappings; the mapping $T$ is said to be Bregman asymptotically quasi-nonexpansive in the intermediate sense if $F(T) \neq \emptyset$ and

$$
\limsup _{n \rightarrow \infty} \sup _{p \in F(T), x \in C}\left(D_{f}\left(p, T^{n} x\right)-D_{f}(p, x)\right) \leq 0 .
$$

Put

$$
\xi_{n}=\max \left\{0, \sup _{p \in F(T), x \in C}\left(D_{f}\left(p, T^{n} x\right)-D_{f}(p, x)\right)\right\} .
$$

This implies $\lim _{n \rightarrow \infty} \xi_{n}=0$. Then (3.1) is reduced to the following:

$$
D_{f}\left(p, T^{n} x\right) \leq D_{f}(p, x)+\xi_{n} \quad \text { for all } p \in F(T) \text { and } x \in C .
$$

Bregman asymptotically quasi-nonexpansive mappings in the intermediate sense are not Lipschitz continuous in general.

Example 3.1 Assume that $E=\mathbf{R}, C=[1 / 2,3 / 2]$ and $T: C \rightarrow C$ defined by

$$
T x= \begin{cases}1, & x \in\left[\frac{1}{2}, 1\right], \\ 1-\sqrt{\frac{x-1}{2},} & x \in\left(1, \frac{3}{2}\right] .\end{cases}
$$

Note that $F(T)=\{1\}$ and $T^{n} x=1$ for all $x \in C$ and $n \geq 2$. If $f: \mathbf{R} \rightarrow(-\infty,+\infty]$ is a Legendre function, then $T$ is Bregman asymptotically quasi-nonexpansive in the intermediate sense since

$$
\limsup _{n \rightarrow \infty} \sup _{x \in C}\left(D_{f}\left(1, T^{n} x\right)-D_{f}(1, x)\right) \leq \limsup _{n \rightarrow \infty} \sup _{x \in C} D_{f}\left(1, T^{n} x\right)=0 .
$$


However, $T$ above is not Lipschitzian with respect to Bregman distances in Example 2.5. Indeed, suppose that there exists $L>0$ such that $D_{f}(T y, T x) \leq L D_{f}(y, x)$ for all $x, y \in C$. By Taylor's theorem, there exists $t \in(0,1)$ such that

$$
D_{f}(y, x)=f(y)-f(x)-\langle\nabla f(x), y-x\rangle=\frac{1}{2} \nabla^{2} f(x+t(y-x))(y-x)^{2} .
$$

(i) Let $f(x)=\|x\|^{2} / 2$ on $\operatorname{dom} f=\mathbf{R}$ and $D_{f}(y, x)=\|y-x\|^{2} / 2$ for all $x, y \in \mathbf{R}$. Put $y=1$ and $x=1+1 / 2(L+1)$. Since $T x=1-1 / 2 \sqrt{L+1}$, we have

$$
\frac{1}{8(L+1)}=\frac{1}{2}\left\|\frac{-1}{2 \sqrt{L+1}}\right\|^{2}=\frac{1}{2}\|T y-T x\|^{2} \leq \frac{L}{2}\|y-x\|^{2}=\frac{L}{8(L+1)^{2}} .
$$

This implies $L+1 \leq L$, which is a contradiction.

(ii) Let $f(x)=x \ln (x)-x$ on $\operatorname{dom} f=[0,+\infty)$ and $D_{f}(y, x)=y \ln (y / x)-y+x$ for all $x \in$ $(0,+\infty)$ and $y \in[0,+\infty)$. Note that $\nabla^{2} f(x)=1 / x$. Put $x=1$. By (3.2), we have

$$
D_{f}(y, 1)=\frac{(y-1)^{2}}{2(1+t(y-1))} \leq \frac{(y-1)^{2}}{2} \text { for } y \geq 1
$$

and

$$
D_{f}(y, 1)=\frac{(y-1)^{2}}{2(1+t(y-1))} \geq \frac{(y-1)^{2}}{2} \quad \text { for } 0<y \leq 1 .
$$

If $y=1+1 / 2(L+1)$, we have

$$
\begin{aligned}
\frac{1}{8(L+1)} & =\frac{1}{2}\left(\frac{-1}{2 \sqrt{L+1}}\right)^{2} \leq D_{f}(T y, 1) \\
& \leq L D_{f}(y, 1) \leq \frac{L}{2}\left(\frac{1}{2(L+1)}\right)^{2}=\frac{L}{8(L+1)^{2}} .
\end{aligned}
$$

This implies $L+1 \leq L$, which is a contradiction.

(iii) Let $f(x)=-\ln (x)$ on $\operatorname{dom} f=(0,+\infty)$ and $D_{f}(y, x)=\ln (x / y)+y / x-1$ for all $x, y \in$ $(0,+\infty)$. Note that $\nabla^{2} f(x)=1 / x^{2}$. Put $y=1$. By (3.2), we have

$$
D_{f}(1, x)=\frac{(1-x)^{2}}{2(x+t(1-x))^{2}} \leq \frac{(1-x)^{2}}{2} \quad \text { for } x \geq 1
$$

and

$$
D_{f}(1, x)=\frac{(1-x)^{2}}{2(x+t(1-x))^{2}} \geq \frac{(1-x)^{2}}{2} \quad \text { for } 0<x \leq 1 .
$$

If $x=1+1 / 2(L+1)$, we have

$$
\begin{aligned}
\frac{1}{8(L+1)} & =\frac{1}{2}\left(\frac{1}{2 \sqrt{L+1}}\right)^{2} \leq D_{f}(1, T x) \\
& \leq L D_{f}(1, x) \leq \frac{L}{2}\left(\frac{-1}{2(L+1)}\right)^{2}=\frac{L}{8(L+1)^{2}} .
\end{aligned}
$$

This implies $L+1 \leq L$, which is a contradiction. 
Theorem 3.2 Let $f: E \rightarrow(-\infty,+\infty]$ be a Legendre function which is totally convex on bounded subsets of E. Suppose that $\nabla f^{*}$ is bounded on bounded subsets of int $\operatorname{dom} f^{*}$. Let $C$ be a nonempty, closed, and convex subset of int $\operatorname{dom} f$. Let $T: C \rightarrow C$ be a closed and Bregman asymptotically quasi-nonexpansive mapping in the intermediate sense. Then $F(T)$ is closed and convex.

Proof Since $T$ is closed, we can easily conclude that $F(T)$ is closed. Now we show the convexity of $F(T)$. Let $p_{1}, p_{2} \in F(T)$ and $p=t p_{1}+(1-t) p_{2}$, where $t \in(0,1)$. We prove that $p \in F(T)$. By the definition of $T$, we have

$$
D_{f}\left(p_{i}, T^{n} p\right) \leq D_{f}\left(p_{i}, p\right)+\xi_{n}
$$

for $i=1,2$. By the three point identity (2.2), we know that

$$
D_{f}(x, y)=D_{f}(x, z)+D_{f}(z, y)+\langle\nabla f(z)-\nabla f(y), x-z\rangle .
$$

This implies

$$
D_{f}\left(p_{i}, T^{n} p\right)=D_{f}\left(p_{i}, p\right)+D_{f}\left(p, T^{n} p\right)+\left\langle\nabla f(p)-\nabla f\left(T^{n} p\right), p_{i}-p\right\rangle
$$

for $i=1$, 2. Combining (3.3) and (3.4) yields

$$
D_{f}\left(p, T^{n} p\right) \leq \xi_{n}-\left\langle\nabla f(p)-\nabla f\left(T^{n} p\right), p_{i}-p\right\rangle
$$

for $i=1$, 2. Multiplying $t$ and $1-t$ on both sides of (3.5) with $i=1$ and $i=2$, respectively, yields

$$
\lim _{n \rightarrow \infty} D_{f}\left(p, T^{n} p\right) \leq \lim _{n \rightarrow \infty}\left(\xi_{n}-\left\langle\nabla f(p)-\nabla f\left(T^{n} p\right), t p_{1}+(1-t) p_{2}-p\right\rangle\right)=0 .
$$

This implies that $\left\{D_{f}\left(p, T^{n} p\right)\right\}_{n \in \mathbf{N}}$ is bounded. By Propositions 2.6 and 2.10, we see that the sequence $\left\{T^{n} p\right\}_{n \in \mathbf{N}}$ is bounded and $\left\|p-T^{n} p\right\| \rightarrow 0$ as $n \rightarrow \infty$. By the closedness of $T$, we have

$$
p=\lim _{n \rightarrow \infty} T^{n+1} p=T\left(\lim _{n \rightarrow \infty} T^{n} p\right)=T p
$$

and hence $p \in F(T)$. Therefore $F(T)$ is convex. This completes the proof.

Theorem 3.2 is reduced to the following results.

Corollary 3.3 ([29], Lemma 1) Let $f: E \rightarrow(-\infty,+\infty]$ be a Legendre function which is totally convex on bounded subsets of $E$. Let $C$ be a nonempty, closed, and convex subset of int $\operatorname{dom} f$ and $T: C \rightarrow C$ a closed and Bergman asymptotically quasi-nonexpansive mapping with the sequence $\left\{k_{n}\right\}_{n \in \mathbf{N}} \subset[1,+\infty)$ such that $k_{n} \rightarrow 1$ as $n \rightarrow \infty$. Then $F(T)$ is closed and convex. 


\section{Main results}

In this section, we prove the following strong convergence theorem for finding a fixed point of a Bregman asymptotically quasi-nonexpansive mapping in the intermediate sense. Let $C$ be a nonempty, closed, and convex subset of $E$ and $T$ a mapping from $C$ into itself. The mapping $T$ is said to be asymptotically regular if, for any $x \in C$,

$$
\lim _{n \rightarrow \infty}\left\|T^{n+1} x-T^{n} x\right\|=0
$$

Theorem 4.1 Let $f: E \rightarrow(-\infty,+\infty]$ be a Legendre function which is bounded, strongly coercive, uniformly Fréchet differentiable and totally convex on bounded subsets on E. Let $C$ be a nonempty, closed, and convex subset of int $\operatorname{dom} f$. Let $T: C \rightarrow C$ be a closed and Bregman asymptotically quasi-nonexpansive mapping in the intermediate sense. Suppose that $T$ is asymptotically regular on $C$ and $F(T)$ is bounded. Let $\left\{x_{n}\right\}$ be a sequence of $C$ generated by

$$
\left\{\begin{array}{l}
x_{0} \in \operatorname{int} \operatorname{dom} f, \quad \text { chosen arbitrarily, } \\
C_{1}=C, \\
x_{1}=\operatorname{proj}_{C}^{f} x_{0}, \\
y_{n}=\nabla f^{*}\left(\alpha_{n} \nabla f\left(x_{n}\right)+\left(1-\alpha_{n}\right) \nabla f\left(T^{n} x_{n}\right)\right), \\
C_{n+1}=\left\{z \in C_{n}: D_{f}\left(z, y_{n}\right) \leq D_{f}\left(z, x_{n}\right)+\xi_{n}\right\}, \\
x_{n+1}=\operatorname{proj}_{C_{n+1}}^{f} x_{0}, \quad n \in \mathbf{N},
\end{array}\right.
$$

where

$$
\xi_{n}=\max \left\{0, \sup _{p \in F(T), x \in C}\left(D_{f}\left(p, T^{n} x\right)-D_{f}(p, x)\right)\right\}
$$

and $\left\{\alpha_{n}\right\}_{n \in \mathbf{N}} \subset[0,1)$ is a sequence satisfying $\lim \sup _{n \rightarrow \infty} \alpha_{n}<1$. Then $\left\{x_{n}\right\}_{n \in \mathbf{N}}$ converges strongly to $\operatorname{proj}_{F(T)}^{f} x_{0}$.

Proof We divide the proof into five steps.

Step 1. We show that $C_{n}$ is closed and convex for all $n \in \mathbf{N}$.

It is obvious that $C_{1}=C$ is closed and convex. Suppose that $C_{m}$ is closed and convex for some $m \in \mathbf{N}$. We see that, for $z \in C_{m}$,

$$
D_{f}\left(z, y_{m}\right) \leq D_{f}\left(z, x_{m}\right)+\xi_{m}
$$

is equivalent to

$$
\left\langle\nabla f\left(x_{m}\right)-\nabla f\left(y_{m}\right), z\right\rangle \leq f^{*}\left(\nabla f\left(x_{m}\right)\right)-f^{*}\left(\nabla f\left(y_{m}\right)\right)+\xi_{m} .
$$

Let $x, y \in C_{m+1}$ and $z=t x+(1-t) y$, where $t \in(0,1)$. By (4.1), we have

$$
\begin{aligned}
& \left\langle\nabla f\left(x_{m}\right)-\nabla f\left(y_{m}\right), z\right\rangle \\
& \quad=t\left\langle\nabla f\left(x_{m}\right)-\nabla f\left(y_{m}\right), x\right\rangle+(1-t)\left\langle\nabla f\left(x_{m}\right)-\nabla f\left(y_{m}\right), y\right\rangle
\end{aligned}
$$




$$
\begin{aligned}
& \leq(t+1-t)\left(f^{*}\left(\nabla f\left(x_{m}\right)\right)-f^{*}\left(\nabla f\left(y_{m}\right)\right)+\xi_{m}\right) \\
& =f^{*}\left(\nabla f\left(x_{m}\right)\right)-f^{*}\left(\nabla f\left(y_{m}\right)\right)+\xi_{m}
\end{aligned}
$$

and hence $z \in C_{m+1}$. Therefore $C_{n}$ is closed and convex for all $n \in \mathbf{N}$. By Proposition 2.11, $\operatorname{proj}_{C_{n}}^{f} x_{0}$ is well defined for all $n \in \mathbf{N}$.

Step 2. We show that $F(T) \subset C_{n}$ for all $n \in \mathbf{N}$.

Let $p \in F(T)$. It is obvious that $F(T) \subset C_{1}=C$. Suppose that $F(T) \subset C_{m}$ for some $m \in \mathbf{N}$. By Proposition 2.7, we have

$$
\begin{aligned}
D_{f}\left(p, y_{m}\right) & =D_{f}\left(p, \nabla f^{*}\left(\alpha_{m} \nabla f\left(x_{m}\right)+\left(1-\alpha_{m}\right) \nabla f\left(T^{m} x_{m}\right)\right)\right) \\
& =W^{f}\left(\alpha_{m} \nabla f\left(x_{m}\right)+\left(1-\alpha_{m}\right) \nabla f\left(T^{m} x_{m}\right), p\right) \\
& \leq \alpha_{m} W^{f}\left(\nabla f\left(x_{m}\right), p\right)+\left(1-\alpha_{m}\right) W^{f}\left(\nabla f\left(T^{m} x_{m}\right), p\right) \\
& =\alpha_{m} D_{f}\left(p, x_{m}\right)+\left(1-\alpha_{m}\right) D_{f}\left(p, T^{m} x_{m}\right) \\
& \leq \alpha_{m} D_{f}\left(p, x_{m}\right)+\left(1-\alpha_{m}\right)\left(D_{f}\left(p, x_{m}\right)+\xi_{m}\right) \\
& \leq D_{f}\left(p, x_{m}\right)+\xi_{m} .
\end{aligned}
$$

This implies $p \in C_{m+1}$. Therefore $F(T) \subset C_{n}$ for all $n \in \mathbf{N}$.

Step 3. We show that $\left\{x_{n}\right\}_{n \in \mathbf{N}}$ is bounded.

Let $p \in F(T)$. By Proposition 2.12(iii), we have

$$
\begin{aligned}
D_{f}\left(x_{n}, x_{0}\right) & =D_{f}\left(\operatorname{proj}_{C_{n}}^{f} x_{0}, x_{0}\right) \\
& \leq D_{f}\left(p, x_{0}\right)-D_{f}\left(p, \operatorname{proj}_{C_{n}}^{f} x_{0}\right) \\
& \leq D_{f}\left(p, x_{0}\right)
\end{aligned}
$$

for all $n \in \mathbf{N}$. This implies that $\left\{D_{f}\left(x_{n}, x_{0}\right)\right\}_{n \in \mathbf{N}}$ is bounded. By Proposition 2.9, the sequence $\left\{x_{n}\right\}_{n \in \mathbf{N}}$ is bounded.

Step 4. We show that every subsequential limit of $\left\{x_{n}\right\}_{n \in \mathbf{N}}$ belongs to $F(T)$.

Since $\left\{x_{n}\right\}_{n \in \mathbf{N}}$ is bounded and $E$ is reflexive, we may assume that $\left\{x_{n_{j}}\right\}_{j \in \mathbf{N}}$ is a weakly convergent subsequence of $\left\{x_{n}\right\}_{n \in \mathbf{N}}$ and denote its weak limit by $\bar{x}$. Since $C_{n}$ is closed and convex, we have $\bar{x} \in C_{n_{j}}$ for all $j \in \mathbf{N}$. By the lower semicontinuity of $f$, we have

$$
\begin{aligned}
D_{f}\left(\bar{x}, x_{0}\right) & =f(\bar{x})-f\left(x_{0}\right)-\left\langle\nabla f\left(x_{0}\right), \bar{x}-x_{0}\right\rangle \\
& \leq \liminf _{j \rightarrow \infty}\left(f\left(x_{n_{j}}\right)-f\left(x_{0}\right)-\left\langle\nabla f\left(x_{0}\right), x_{n_{j}}-x_{0}\right\rangle\right) \\
& =\liminf _{j \rightarrow \infty} D_{f}\left(x_{n_{j}}, x_{0}\right) \\
& \leq \limsup _{j \rightarrow \infty} D_{f}\left(x_{n_{j}}, x_{0}\right) \leq D_{f}\left(\bar{x}, x_{0}\right) .
\end{aligned}
$$

This implies

$$
\lim _{j \rightarrow \infty} D_{f}\left(x_{n_{j}}, x_{0}\right)=D_{f}\left(\bar{x}, x_{0}\right) .
$$


By Proposition 2.12(iii), we have

$$
\begin{aligned}
\lim _{j \rightarrow \infty} D_{f}\left(\bar{x}, x_{n_{j}}\right) & =\lim _{j \rightarrow \infty} D_{f}\left(\bar{x}, \operatorname{proj}_{C_{n_{j}}}^{f} x_{0}\right) \\
& \leq \lim _{j \rightarrow \infty}\left(D_{f}\left(\bar{x}, x_{0}\right)-D_{f}\left(\operatorname{proj}_{C_{n_{j}}}^{f} x_{0}, x_{0}\right)\right) \\
& =\lim _{j \rightarrow \infty}\left(D_{f}\left(\bar{x}, x_{0}\right)-D_{f}\left(x_{n_{j}}, x_{0}\right)\right)=0 .
\end{aligned}
$$

By Proposition 2.10, we have $\left\|\bar{x}-x_{n_{j}}\right\| \rightarrow 0$ as $j \rightarrow \infty$. By Proposition 2.2, we have

$$
\lim _{j \rightarrow \infty}\left\|\nabla f(\bar{x})-\nabla f\left(x_{n_{j}}\right)\right\|=0
$$

Since $x_{n}=\operatorname{proj}_{C_{n}}^{f} x_{0} \in C_{n}$ and $x_{n+1}=\operatorname{proj}_{C_{n+1}}^{f} x_{0} \in C_{n+1} \subset C_{n}$, we have $D_{f}\left(x_{n}, x_{0}\right) \leq$ $D_{f}\left(x_{n+1}, x_{0}\right)$. This implies that $\left\{D_{f}\left(x_{n}, x_{0}\right)\right\}_{n \in \mathbf{N}}$ is nondecreasing and the limit of $D_{f}\left(x_{n}, x_{0}\right)$ exists as $n \rightarrow \infty$. By Proposition 2.12(iii), we have

$$
\begin{aligned}
D_{f}\left(x_{n+1}, x_{n}\right) & =D_{f}\left(x_{n+1}, \operatorname{proj}_{C_{n}}^{f} x_{0}\right) \\
& \leq D_{f}\left(x_{n+1}, x_{0}\right)-D_{f}\left(\operatorname{proj}_{C_{n}}^{f} x_{0}, x_{0}\right) \\
& =D_{f}\left(x_{n+1}, x_{0}\right)-D_{f}\left(x_{n}, x_{0}\right)
\end{aligned}
$$

for all $n \in \mathbf{N}$. This implies

$$
\lim _{n \rightarrow \infty} D_{f}\left(x_{n+1}, x_{n}\right)=0
$$

By Proposition 2.10, we have $\left\|x_{n+1}-x_{n}\right\| \rightarrow 0$ as $n \rightarrow \infty$. By Proposition 2.2, we have

$$
\lim _{n \rightarrow \infty}\left\|\nabla f\left(x_{n+1}\right)-\nabla f\left(x_{n}\right)\right\|=0
$$

Since $x_{n+1} \in C_{n+1}$, we have $D_{f}\left(x_{n+1}, y_{n}\right) \leq D_{f}\left(x_{n+1}, x_{n}\right)+\xi_{n}$ for all $n \in \mathbf{N}$. By (4.5), we have $D_{f}\left(x_{n+1}, y_{n}\right) \rightarrow 0$ as $n \rightarrow \infty$. By Proposition 2.10, we have $\left\|x_{n+1}-y_{n}\right\| \rightarrow 0$ as $n \rightarrow \infty$. By Proposition 2.2, we have

$$
\lim _{n \rightarrow \infty}\left\|\nabla f\left(x_{n+1}\right)-\nabla f\left(y_{n}\right)\right\|=0
$$

By the definition of $y_{n}$, we have

$$
\begin{aligned}
& \left\|\nabla f\left(T^{n} x_{n}\right)-\nabla f\left(x_{n+1}\right)\right\| \\
& \quad \leq \frac{1}{1-\alpha_{n}}\left\|\nabla f\left(x_{n+1}\right)-\nabla f\left(y_{n}\right)\right\|+\frac{\alpha_{n}}{1-\alpha_{n}}\left\|\nabla f\left(x_{n+1}\right)-\nabla f\left(x_{n}\right)\right\| .
\end{aligned}
$$

By (4.6) and (4.7), we find from $\limsup _{n \rightarrow \infty} \alpha_{n}<1$ that

$$
\lim _{n \rightarrow \infty}\left\|\nabla f\left(T^{n} x_{n}\right)-\nabla f\left(x_{n+1}\right)\right\|=0
$$

We have

$$
\left\|\nabla f\left(T^{n} x_{n}\right)-\nabla f(\bar{x})\right\| \leq\left\|\nabla f\left(T^{n} x_{n}\right)-\nabla f\left(x_{n+1}\right)\right\|+\left\|\nabla f\left(x_{n+1}\right)-\nabla f(\bar{x})\right\| .
$$


By (4.4) and (4.8), we have $\left\|\nabla f\left(T^{n_{j}} x_{n_{j}}\right)-\nabla f(\bar{x})\right\| \rightarrow 0$ as $j \rightarrow \infty$. By Propositions 2.3 and 2.8, $\nabla f^{*}$ is uniformly continuous on bounded subsets of $E^{*}$ and thus $\left\|T^{n_{j}} x_{n_{j}}-\bar{x}\right\| \rightarrow 0$ as $j \rightarrow \infty$. Since $f$ is asymptotically regular, we have

$$
\lim _{j \rightarrow \infty}\left\|T^{n_{j}+1} x_{n_{j}}-\bar{x}\right\| \leq \lim _{j \rightarrow \infty}\left(\left\|T^{n_{j}+1} x_{n_{j}}-T^{n_{j}} x_{n_{j}}\right\|+\left\|T^{n_{j}} x_{n_{j}}-\bar{x}\right\|\right)=0 .
$$

This implies $T T^{n_{j}} x_{n_{j}}-\bar{x} \rightarrow 0$ as $j \rightarrow \infty$. By the closedness of $T$, we have $\bar{x}=T \bar{x}$. Therefore, the limit of $\left\{x_{n_{j}}\right\}_{j \in \mathbf{N}}$ belongs to $F(T)$.

Step 5. We show that $x_{n} \rightarrow \operatorname{proj}_{F(T)}^{f} x_{0}$ as $n \rightarrow \infty$.

Since $\operatorname{proj}_{F(T)}^{f} x_{0} \in F(T) \subset C_{n}$ and $x_{n}=\operatorname{proj}_{C_{n}}^{f} x_{0}$, we have $D_{f}\left(x_{n}, x_{0}\right) \leq D_{f}\left(\operatorname{proj}_{F(T)}^{f} x_{0}, x_{0}\right)$ for all $n \in \mathbf{N}$. By (4.3), we have

$$
D_{f}\left(\bar{x}, x_{0}\right)=\lim _{j \rightarrow \infty} D_{f}\left(x_{n_{j}}, x_{0}\right) \leq D_{f}\left(\operatorname{proj}_{F(T)}^{f} x_{0}, x_{0}\right)
$$

Thus $\bar{x}=\operatorname{proj}_{F(T)}^{f} x_{0}$ since $\bar{x} \in F(T)$. Hence $\bar{x}$ is only strong cluster point of $x_{n}$. Therefore $x_{n} \rightarrow \operatorname{proj}_{F(T)}^{f} x_{0}$ as $n \rightarrow \infty$. This completes the proof.

$$
\text { If } f(x)=\|x\|^{2} / 2 \text { for all } x \in E \text {, then Theorem } 4.1 \text { is reduced to the following corollary. }
$$

Corollary 4.2 ([11], Theorem 2.1) Let E be a reflexive, strictly convex and smooth Banach space such that both $E$ and $E^{*}$ have the Kadec-Klee property. Let $C$ be a nonempty, closed, and convex subset of E. Let $T: C \rightarrow C$ be an asymptotically quasi- $\phi$-nonexpansive mapping in the intermediate sense. Assume that $T$ is asymptotically regular on $C$ and closed, and $F(T) \neq \emptyset$ is bounded. Let $\left\{x_{n}\right\}$ be a sequence generated by

$$
\left\{\begin{array}{l}
x_{0} \in E, \quad \text { chosen arbitrarily, } \\
C_{1}=C \\
x_{1}=\Pi_{C_{1} x_{0}} \\
y_{n}=J^{-1}\left(\alpha_{n} J x_{n}+\left(1-\alpha_{n}\right) J T^{n} x_{n}\right), \\
C_{n+1}=\left\{z \in C_{n}: \phi\left(z, y_{n}\right) \leq \phi\left(z, x_{n}\right)+\xi_{n}\right\} \\
x_{n+1}=\Pi_{C_{n+1}} x_{1}, \quad n \in \mathbf{N},
\end{array}\right.
$$

where

$$
\xi_{n}:=\max \left\{0, \sup _{p \in F(T), x \in C}\left(\phi\left(p, T^{n} x\right)-\phi(p, x)\right)\right\}
$$

$\Pi_{C_{n}}$ is the generalized projection from $E$ onto $C_{n}$ and $\left\{\alpha_{n}\right\}_{n \in \mathbf{N}} \subset[0,1)$ is a sequence satisfying limsup $\operatorname{sum}_{n \rightarrow \infty} \alpha_{n}<1$. Then $\left\{x_{n}\right\}_{n \in \mathbf{N}}$ converges strongly to $\Pi_{F(T)} x_{1}$, where $\Pi_{F(T)}$ is the generalized projection from $C$ onto $F(T)$.

Proof Using the technique used in the proof of Theorem 4.1 with $f(x)=\|x\|^{2} / 2$ for all $x \in E$, we find that the sequence $\left\{x_{n}\right\}$ generated by (4.9) converges strongly to $\Pi_{F(T)} x_{1}$. 


\section{Competing interests}

The author declares that she has no competing interests.

\section{Received: 10 January 2014 Accepted: 13 June 2014 Published: 22 Jul 2014}

\section{References}

1. Goebel, K, Kirk, WA: A fixed point theorem for asymptotically nonexpansive mappings. Proc. Am. Math. Soc. 35, 171-174 (1972)

2. Takahashi, W, Takeuchi, Y, Kubota, R: Strong convergence theorems by hybrid methods for families of nonexpansive mappings in Hilbert spaces. J. Math. Anal. Appl. 341, 276-286 (2008)

3. Schu, J: Weak and strong convergence to fixed points of asymptotically nonexpansive mappings. Bull. Aust. Math. Soc. 43, 153-159 (1991)

4. Inchan, I: Strong convergence theorems of modified Mann iteration methods for asymptotically nonexpansive mappings in Hilbert spaces. Int. J. Math. Anal. 2, 1135-1145 (2008)

5. Bruck, RE, Kuczumow, T, Reich, S: Convergence of iterates of asymptotically nonexpansive mappings in Banach spaces with the uniform Opial property. Colloq. Math. 65, 169-179 (1993)

6. Hecai, Y, Aichao, L: Projection algorithms for treating asymptotically quasi- $\phi$-nonexpansive mappings in the intermediate sense. J. Inequal. Appl. (2013). doi:10.1186/1029-242X-2013-265

7. Qing, Y: Some results on asymptotically quasi- $\phi$-nonexpansive mappings in the intermediate sense. J. Fixed Point Theory 2012, Article ID 1, (2012)

8. Qin, X, Huang, S, Wang, T: On the convergence of hybrid projection algorithms for asymptotically quasi- $\phi$-nonexpansive mappings. Comput. Math. Appl. 61, 851-859 (2011)

9. Qin, X, Wang, L: On asymptotically quasi- $\phi$-nonexpansive mappings in the intermediate sense. Abstr. Appl. Anal. 2012, Article ID 636217 (2012). doi:10.1155/2012/636217

10. Alber, Yl: Metric and generalized projection operators in Banach spaces: properties and applications. In: Kartsatos, AG (ed.) Theory and Applications of Nonlinear Operators of Accretive and Monotone Type. Lecture Notes Pure Appl. Math., vol. 178, pp. 15-50. Dekker, New York (1996)

11. Hao, Y: Some results on a modified Mann iterative scheme in a reflexive Banach space. Fixed Point Theory Appl. 2013 Article ID 227 (2013). doi:10.1186/1687-1812-2013-227

12. Bregman, LM: The relaxation method of finding the common point of convex sets and its application to the solution of problems in convex programming. USSR Comput. Math. Math. Phys. 7, 200-217 (1967)

13. Martin-Marquez, V, Reich, S, Sabach, S: Iterative methods for approximating fixed points of Bregman nonexpansive operators. Discrete Contin. Dyn. Syst. 6, 1043-1063 (2013)

14. Reich, S, Sabach, S: Two strong convergence theorems for Bregman strongly nonexpansive operators in reflexive Banach spaces. Nonlinear Anal. 73, 122-135 (2010)

15. Reich, S, Sabach, S: Existence and approximation of fixed points of Bregman firmly nonexpansive mappings in reflexive Banach spaces. In: Fixed-Point Algorithms for Inverse Problems in Science and Engineering. Springer Optim. Appl., vol. 49, pp. 301-316. Springer, New York (2011)

16. Suantai, S, Cho, YJ, Cholamjiak, P: Halpern's iteration for Bregman strongly nonexpansive mappings in reflexive Banach spaces. Comput. Math. Appl. 64, 489-499 (2012)

17. Bauschke, HH, Borwein, JM, Combettes, PL: Essential smoothness, essential strict convexity, and Legendre functions in Banach spaces. Commun. Contemp. Math. 3, 615-647 (2001)

18. Barbu, V, Precupanu, T: Convexity and Optimization in Banach Spaces. Springer, Dordrecht (2012)

19. Ambrosetti, A, Prodi, G: A Primer of Nonlinear Analysis. Cambridge University Press, Cambridge (1993)

20. Reich, S, Sabach, S: A strong convergence theorem for a proximal-type algorithm in reflexive Banach spaces. J. Nonlinear Convex Anal. 10, 471-485 (2009)

21. Zălinescu, C: Convex Analysis in General Vector Spaces. World Scientific, River Edge (2002)

22. Butnariu, D, lusem, AN: Totally Convex Functions for Fixed Points Computation and Infinite Dimensional Optimization. Kluwer Academic, Dordrecht (2000)

23. Censor, Y, Lent, A: An iterative row-action method for interval convex programming. J. Optim. Theory Appl. 34, 321-353 (1981)

24. Chen, $\mathrm{G}$, Teboulle, M: Convergence analysis of a proximal-like minimization algorithm using Bregman functions. SIAM J. Optim. 3, 538-543 (1993)

25. Butnariu, D, lusem, AN, Zălinescu, C: On uniform convexity, total convexity and convergence of the proximal point and outer Bregman projection algorithms in Banach spaces. J. Convex Anal. 10, 35-61 (2003)

26. Butnariu, D, Resmerita, E: Bregman distances, totally convex functions, and a method for solving operator equations in Banach spaces. Abstr. Appl. Anal. 2006, Article ID 84919 (2006)

27. Reich, S, Sabach, S: Two strong convergence theorems for a proximal method in reflexive Banach spaces. Numer. Funct. Anal. Optim. 31, 22-44 (2010)

28. Alber, Y, Butnariu, D: Convergence of Bregman projection methods for solving consistent convex feasibility problems in reflexive Banach spaces. J. Optim. Theory Appl. 92, 33-61 (1997)

29. Wang, S, Kang, SM: Strong convergence iterative algorithms for equilibrium problems and fixed point problems in Banach spaces. Abstr. Appl. Anal. 2013, Article ID 619762 (2013) 